\title{
Characterization of the $T$ cell repertoire by deep $T$ cell receptor sequencing in tissues and blood from patients with advanced colorectal cancer
}

\author{
KENJI TAMURA ${ }^{1}$, SHOICHI HAZAMA ${ }^{2}$, RUI YAMAGUCHI ${ }^{3}$, SEIYA IMOTO $^{3}$, HIROKO TAKENOUCHI ${ }^{2}$, \\ YUKA INOUE $^{2}$, SHINSUKE KANEKIYO ${ }^{2}$, YOSHITARO SHINDO ${ }^{2}$, SATORU MIYANO $^{3}$, \\ YUSUKE NAKAMURA $^{1}$ and KAZUMA KIYOTANI ${ }^{1}$
}

\author{
${ }^{1}$ Section of Hematology/Oncology, Department of Medicine, The University of Chicago, Chicago, IL 60637, USA; \\ ${ }^{2}$ Department of Digestive Surgery and Surgical Oncology, Yamaguchi University Graduate School of Medicine, Ube, \\ Yamaguchi 755-8505; ${ }^{3}$ Human Genome Center, Institute of Medical Science, The University of Tokyo, Tokyo 108-8639, Japan
}

Received December 13, 2014; Accepted January 21, 2016

DOI: $10.3892 / \mathrm{ol} .2016 .4465$

\begin{abstract}
The aim of the present study was to characterize infiltrated $\mathrm{T}$ cell clones that define the tumor immune environment and are important in the response to treatment in patients with advanced colorectal cancer (CRC). In order to explore predictive biomarkers for the efficacy of immunochemotherapies, $\mathrm{T}$ cell receptor (TCR) repertoire analysis was performed using blood samples and tumor tissues obtained from patients with advanced CRC that had been treated with a combination of five-cancer peptide vaccines and oxaliplatin-based chemotherapy. The TCR- $\alpha / \beta$ complementary DNAs (cDNAs), prepared from the messenger RNAs (mRNAs) obtained from 17 tumor tissues and 39 peripheral blood mononuclear cells of 9 CRC patients at various time points, were sequenced. The oligoclonal enrichment of certain TCR sequences was identified in tumor tissues and blood samples; however, only a few TCR sequences with a frequency of $>0.1 \%$ were commonly detected in pre- and post-treatment tumor tissues, or in post-treatment blood and tissue samples. The average correlation coefficients of the TCR- $\alpha$ and TCR- $\beta$ clonotype frequencies between the post-treatment tumor tissues and blood samples were 0.023 and 0.035 , respectively, and were much lower compared with the correlation coefficients of the TCR- $\alpha$ and TCR- $\beta$ clonotype frequencies between pre- and post-treatment blood samples ( 0.430 and 0.370 , respectively), suggesting that $\mathrm{T}$ cell populations in tumor tissues vary from those in blood. Although the sample size was small, a tendency for the TCR diversity in tumor tissues to drastically decrease during the treatment was indicated in two patients, who
\end{abstract}

Correspondence to: Professor Yusuke Nakamura, Section of Hematology/Oncology, Department of Medicine, The University of Chicago, 900 E. 57th Street, Chicago, IL 60637, USA

E-mail: ynakamura@bsd.uchicago.edu

Key words: colorectal cancer, $\mathrm{T}$ cell receptor repertoire, TCR- $\alpha$, TCR- $\beta$, diversity, clonotype, predictive biomarker exhibited a longer progression-free survival time. The results of the present study suggest that TCR diversity scores in tissues may be a useful predictive biomarker for the therapeutic effect of immunochemotherapy for patients with advanced CRC.

\section{Introduction}

Accumulating data have indicated the significance of anti-tumor immune responses in the prognosis of patients with various types of cancer $(1,2)$. Tumor infiltrating lymphocytes (TILs) were shown to be associated with the prognoses of patients with colorectal cancer (CRC), and are also considered to be important as a predictor for the therapeutic response to chemotherapy and radiotherapy (3-7). Although immunotherapy using peptide vaccines is expected to be a promising method to treat cancer, it is not established as a treatment modality due to its lack of efficacy. Several mechanisms to protect cancer cells from host immune attacks in tumor tissues have been proposed (8-10). For example, the limitation of anti-tumor effects by cytotoxic T lymphocytes was partly explained by: i) Downregulation or loss of expression of human leukocyte antigen (HLA) or targeted antigen proteins; ii) upregulation of programmed death-ligand 1 by tumor cells; iii) suppression of immune responses by production of the tryptophan catabolic enzyme indoleamine 2,3-dioxygenase by dendritic cells and macrophages; iv) accumulation of regulatory T cells; v) loss of the costimulatory molecules cluster of differentiation CD80 and CD86, which are ligands of CD28 and essential in inducing immune responses; and vi) production of strong immunosuppressive cytokines, including interleukin (IL)-6, IL-10 and transforming growth factor- $\beta$. TILs are one of the key factors that define the tumor microenvironment and may act as a predictive biomarker for the response to immunotherapy. However, the majority of previous studies focus on the quantification of the number of TILs or CD8 ${ }^{+}$cells in tumors, without an examination of the functional or clonal characteristics that confer to anti-tumor immune effects (3-7). Characterizing the $\mathrm{T}$ cell repertoire in detail is essential to improve the understanding of the tumor microenvironment 
that is associated with the clinical responses to various cancer therapies. In addition, monitoring the immune responses in cancer patients during the treatment is also important.

In humans, the majority ( 95\%) of T lymphocytes carry $\mathrm{T}$ cell receptors (TCRs), which consists of a heterodimer of an $\alpha$-chain and a $\beta$-chain (11). The high degree of the diversity in TCRs is generated by a somatic recombination process of variable (V), diversity (D) (only for $\beta$-chain) and joining $(\mathrm{J})$ exons, termed the $\mathrm{V}(\mathrm{D}) \mathrm{J}$ recombination (11). Random trimming and the addition of non-template nucleotides at the junction site significantly increase the TCR diversity. The rearrangement of the V, (D) and J segments defines the highly variable complementarity determining region 3 (CDR3), which is critical to characterize the antigen recognition specificity of individual $\mathrm{T}$ cell clones (12). A repertoire of $10^{15-18}$ various TCRs may be generated in humans $(13,14)$. Therefore, the characterization of the TCR in TILs may reveal important information regarding the biologically important anti-tumor T cell responses (15). In the present study, the $\mathrm{T}$ cell repertoire was analyzed in blood samples and cancer tissues in advanced CRC patients that had received cancer vaccine treatment, with the aim of exploring predictive biomarkers for the efficacy prior to treatment, and for selecting of patients that are likely to exhibit better clinical outcomes.

\section{Materials and methods}

Patients. Due to the availability of surgical specimens, 9 out of 96 patients with advanced CRC that were enrolled in a previous phase II study (FXV study)were selected for the present study (16). All patients in the present study were enrolled at Yamaguchi University Hospital (Ube, Japan) between February 2009 and November 2012. A total of 17 tumor tissues from the surgical resection of primary or metastatic CRCs and 39 blood samples were collected, as summarized in Table I. Pre- and post-treatment tumor tissues were collected from 6 patients. Blood samples were collected at various time points, and peripheral blood mononuclear cells (PBMCs) were isolated from whole blood using Ficoll-Paque PLUS (GE Healthcare Bio-Sciences, Pittsburgh, PA, USA). The present study was approved by the Institutional Review Boards of the University of Chicago (Chicago, IL, USA; approval no., 13-0797) and Yamaguchi University (Ube, Japan; approval no., H23-135).

Library preparation. The libraries for TCR sequencing were prepared according to a previously established method (17). Total RNA was extracted from tumor tissues and PBMCs using an RNeasy Mini kit (Qiagen, Inc., Valencia, CA, USA) with DNase treatment. Complementary DNA (cDNA) with the 5'-RACE adapter was synthesized using the SMART cDNA Library Construction kit and Advantage 2 Polymerase (Clontech Laboratories, Inc., Mountainview, CA, USA) following the manufacturer's protocol. Second-round polymerase chain reaction (PCR) was performed using the Platinum PCR SuperMix High Fidelity (Thermo Fisher Scientific, Waltham, MA, USA) with a forward fusion-primer, consisting of an Ion Torrent trP1 adaptor sequence and 5' universal primer sequence, and a reverse fusion-primer consisting of an Ion Torrent $\mathrm{A}$ adaptor and specific sequence to the $\mathrm{C}$ region of the TCR- $\alpha$ or TCR- $\beta$ (17). The amplification thermocycle consisted of: $3 \mathrm{~min}$ at $94^{\circ} \mathrm{C}$; and 40 cycles of $30 \mathrm{sec}$ at $94^{\circ} \mathrm{C}, 30 \mathrm{sec}$ at $65^{\circ} \mathrm{C}$ and $1 \mathrm{~min}$ at $68^{\circ} \mathrm{C}$; followed by purification using AMPure XP (Beckman Coulter, Inc., Brea, CA, USA) to obtain a final library. The sequencing templates were prepared on the OneTouch2 system and subjected to DNA sequencing on the Ion PGM sequencer using the Ion PGM Sequencing 400 kit and Ion 318 Chip kit v2 (Thermo Fisher Scientific).

Sequencing analysis. Raw fastq files were analyzed using Tcrip software (17). Briefly, each of the reads was separately mapped to the International ImMunoGeneTics reference sequences of the V, J and constant (C) gene segments of TCR- $\alpha$ or TCR- $\beta$ using the Bowtie 2 aligner, version 2.1.0 (18,19). Among the reads that were properly mapped to the $\mathrm{V}, \mathrm{J}$ and $\mathrm{C}$ segments, a junction sequence between the $\mathrm{V}$ and $\mathrm{J}$ segments in the read was analyzed for TCR- $\alpha$ and TCR- $\beta$. For TCR- $\alpha$, the junction sequence was recognized as an $\mathrm{N}$ segment. For TCR- $\beta$, a $\mathrm{D}$ segment in the junction sequence was searched by scoring similarities of sub-sequences of the junction sequences with the reference sequences of D segments using a sliding window method (20), and then N1 and N2 segments were determined. Following the decomposition of reads into the V, D, J and $\mathrm{C}$ segments, the amino acid sequences of the CDR3 regions were determined, starting with the second conserved cysteine in the $\mathrm{V}$ segment and ending with the conserved phenylalanine in the $\mathrm{J}$ segment.

Statistical analysis. Correlation coefficient and TCR diversity was compared using the Student's $t$-test. $\mathrm{P}<0.05$ was considered to indicate a statistically significant difference. Pearson's correlation was used for correlation analysis All statistical analyses were performed using the $\mathrm{R}$ statistical environment version 2.15.2 (available from, www.r-project.org).

\section{Results}

TCR sequencing of tumor and blood samples from advanced $C R C$ patients. The sequences of TCR- $\alpha$ and TCR- $\beta$ cDNAs were prepared from a total of 56 samples (17 tumor tissue samples and 39 blood samples) at different time points, which were obtained from 9 patients with advanced CRC that were treated with cancer vaccines, were analyzed (Table I). Through cDNA sequencing of TCR, on average 550,116 $\pm 422,311$ and $215,160 \pm 243,900$ reads mapped to V(D)J and C segments were obtained for TCR- $\alpha$ and TCR- $\beta$, respectively. From the analysis of these reads, 32,608 $\pm 28,216$ unique clonotypes for TCR- $\alpha$ and 28,693 $\pm 29,361$ unique clonotypes for TCR- $\beta$ were identified. Fig. 1A-D shows the representative results of patient CRC6. Enrichment of certain TCR sequences was observed in the tumor tissues and blood. However, the majority of enriched sequences with a frequency of $>0.5 \%$ in pre- or post-treatment tumor samples were not commonly detected in these samples, and were not identified in TCR sequence reads in the blood samples of the same patients (Fig. 1A and B).

By applying the correlation analysis, a strong correlation was indicated in the TCR clonotypes among pre- and post-treatment blood samples (correlation coefficients, $r=0.83$ and 0.82 for TCR- $\alpha$ and TCR- $\beta$, respectively), but no correlation between pre- and post-treatment tumor tissues ( $r=0.001$ and 0.006 , respectively), or between tumor tissues and blood samples 
Tumor

Pre (-4 weeks)

- Post (44 weeks)

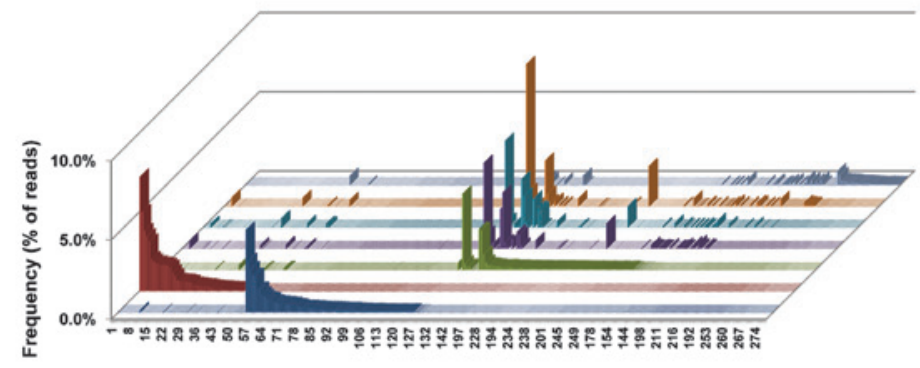

Blood

Pre ( 0 week)

- Post ( 5 weeks)

- Post (19 weeks)

- Post (35 weeks)

Post (77 weeks)

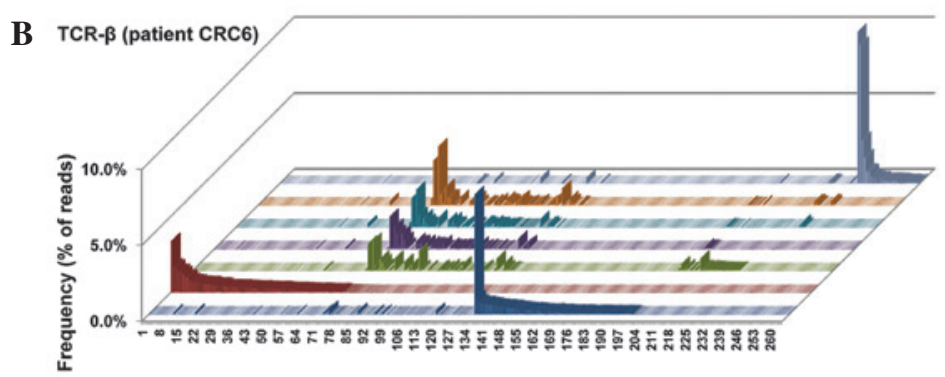

C TCR- $\alpha$ (patient CRC6)
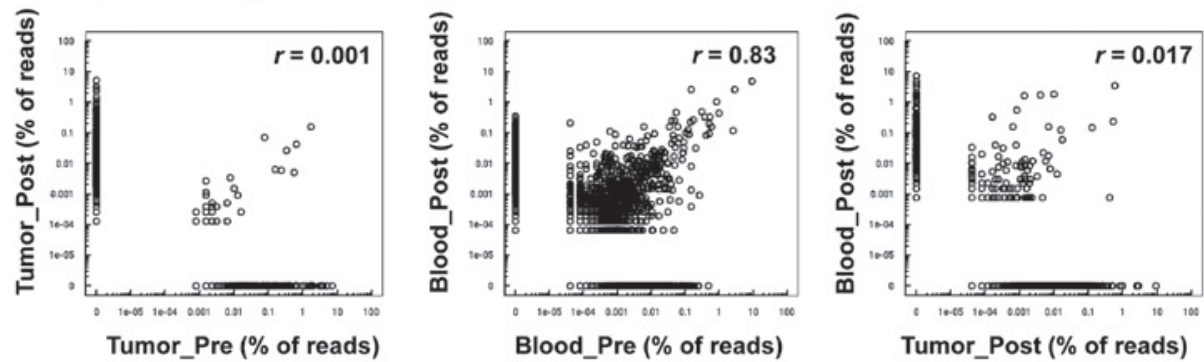

D TCR- $\beta$ (patient CRC6)
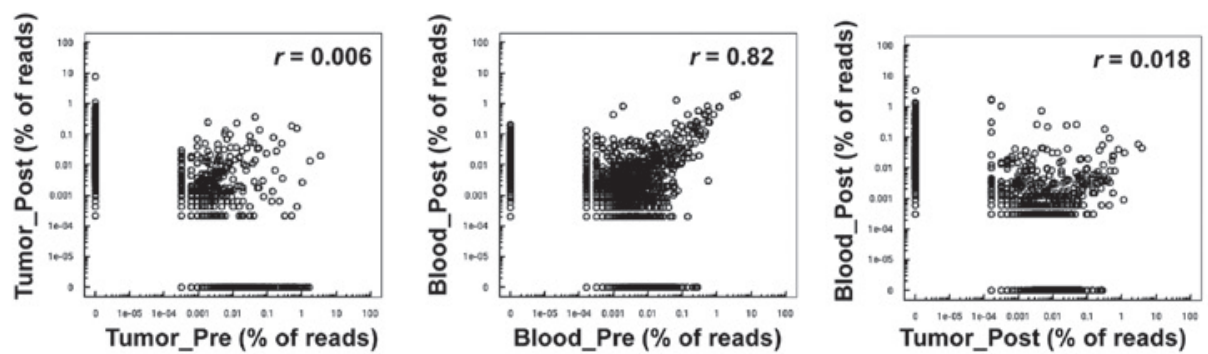

$\mathbf{E}$
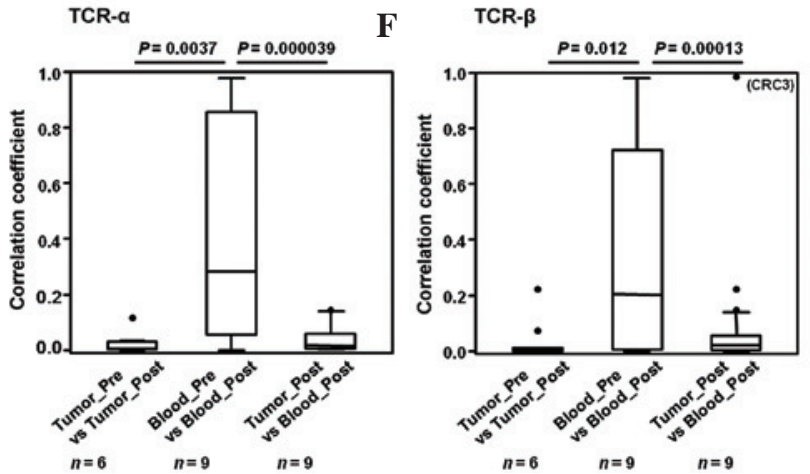

Figure 1. Distribution of the TCR clonotype in tumor tissues and blood of 9 patients with CRC. Frequency distribution of clonotypes of (A) TCR- $\alpha$ and (B) TCR- $\beta$ in tumor tissues and blood samples from patient CRC6. Clonotypes with functional CDR3 identified at $>0.1 \%$ in at least one sample were plotted. The frequency was calculated based on the total reads, which include non-functional CDR3. Clonotype frequency plots of (C) TCR- $\alpha$ and (D) TCR- $\beta$ in patient CRC6. Each dot on the scatter plots indicates a single clonotype with a normalized $\log _{10}$ clone count graphed at pre-treatment tumor tissue vs. post-treatment tumor tissue (left), pre-treatment blood vs. post-treatment blood (middle) and post-treatment tumor tissue vs. post-treatment blood (right). Blood sample at 35 weeks was used as post-treatment blood. Comparison of the correlation coefficient calculated in (E) TRC- $\alpha$ and (F) TRC- $\beta$ clonotype frequency plots of 9 patients with CRC. The horizontal line indicates the median concentration, the box covers the 25-75 percentiles and the maximum length of each whisker is 1.5 times the interquartile range. Each dot indicates an outlier. TRC, T cell receptor; CRC, colorectal cancer; CDR3, complementarity determining region 3. 
G
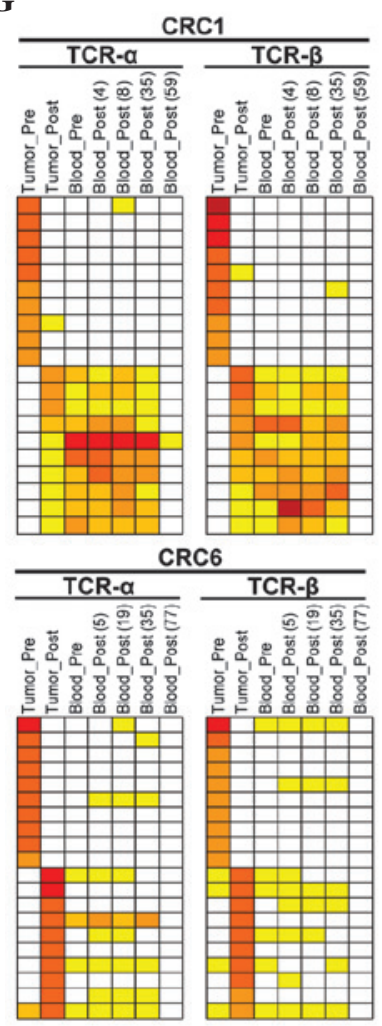
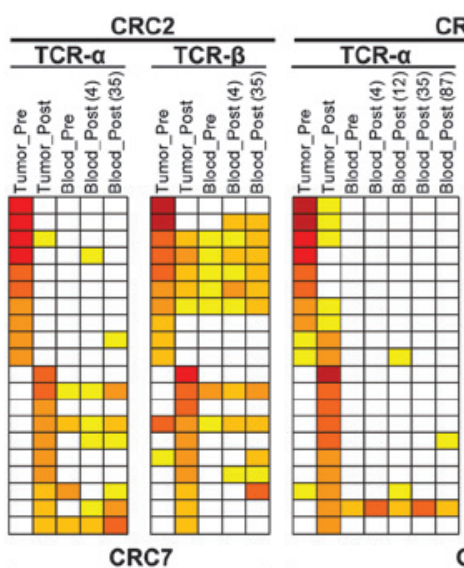

CRC3
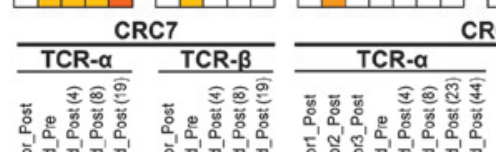
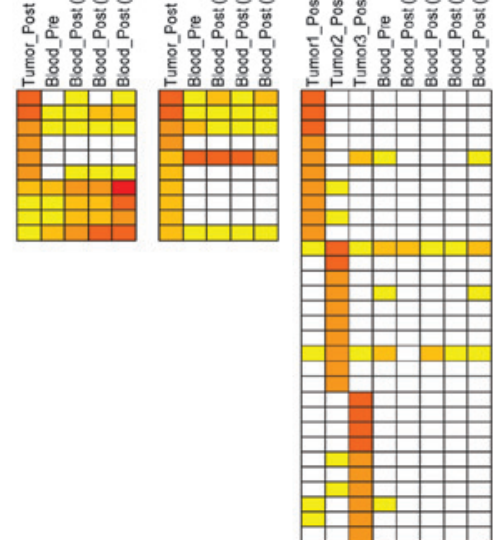
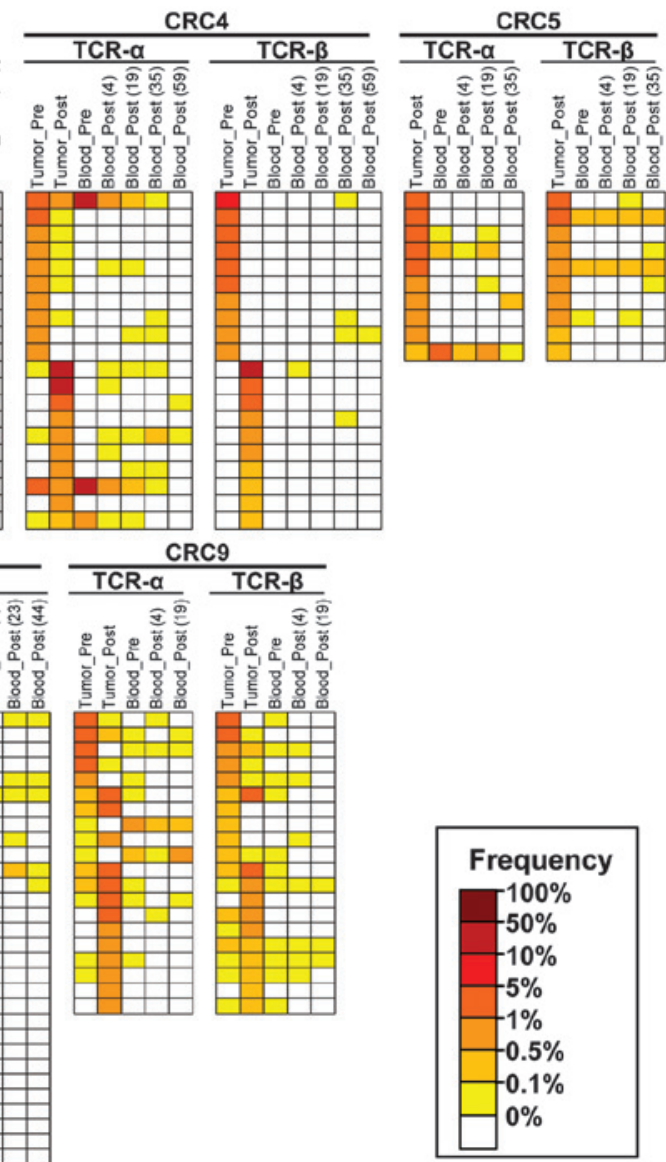

Figure 1. Continued. (G) Heatmaps of TCR clonotype frequency in tumor tissues and blood samples. The 10 most abundant TCR clonotypes in each of the pre- and post-treatment tumor samples are selected. The vertical axis indicates TCR clonotype. The number in brackets indicates the timing of sample collection from the initiation of immunochemotherapy. TCR, T cell receptor; CRC, colorectal cancer.

$(r=0.017$ and 0.018 , respectively) were indicated (Fig. 1C and D). Similarly, the median correlation coefficients for comparing between the pre- and post-treatment tissue samples $(n=6)$, were 0.006 and 0.002 for TCR- $\alpha$ and TCR- $\beta$, respectively, and 0.016 and 0.021 for TCR- $\alpha$ and TCR- $\beta$, respectively, in the tumor tissue and blood comparison $(n=9)$, which were much smaller compared with the coefficients in the pre- and post-treatment blood comparison $(n=9 ; 0.28$ and 0.20 for TCR- $\alpha$ and TCR- $\beta$, respectively) (Fig. 1E and F).

The heat map in Fig. 1G indicates that TCR sequences enriched in pre-treatment tumor tissues were undetectable in blood samples; but, notably, TCR sequences enriched in the post-treatment tumor tissues were detectable in the blood samples, although their frequencies were as low as $0.1-0.5 \%$. Particularly, patient CRC3 showed a higher correlation coefficient between $\mathrm{T}$ cells in a post-treatment tumor tissue and blood sample, which shared an abundant TCR- $\beta$ clonotype with TRBV7-2, TRBJ2-7 and CDR3 (sequence, CASSLDPGWTYEQYF). These results indicate that, although TCR clonotypes in tumor tissues are almost entirely different from those in blood samples, several TCR clonotypes enriched in tumor tissues were commonly observed in circulating lymphocytes during the treatment.

Association between TCR diversity and clinical outcome. The TCR diversity was calculated using the inverse Simpson's diversity index $\left(1 / D_{\mathrm{s}}\right)(21)$, and the association between the TCR diversity and patients' clinical outcome was investigated. The 9 CRC patients were divided into two groups based on progression-free survival (PFS), consisting of the favorable (PFS $\geq 12$ months) and unfavorable (PFS $<12$ months) groups, and then the TCR diversity between these two groups was compared (Fig. 2A and B). A tendency for lower diversity scores of TCR- $\alpha$ and TCR- $\beta$ was indicated in post-treatment tumor tissues (TCR- $\alpha, \mathrm{P}=0.16$; TCR- $\beta, \mathrm{P}=0.14$; Fig. $2 \mathrm{C}$ and D). A decrease in TCR diversity during treatment was also observed in the favorable group compared with the unfavorable group (TCR- $\alpha, \mathrm{P}=0.19$; TCR- $\beta, \mathrm{P}=0.29$; Fig. $2 \mathrm{E}$ and F). In blood samples, the higher ratio of TCR- $\beta$ diversity at the early time point (8-19 weeks subsequent to the initiation of treatment) relative to pre-treatment tended to associate with a longer PFS; in particular, the greatest ratio was observed in patient CRC3, who exhibited the longest PFS (Fig. 3A and B). Notably, the diversity of TCR- $\alpha$ and TCR- $\beta$ in blood samples was markedly decreased following the surgical resection of tumors in two patients (CRC2 and CRC7).

\section{Discussion}

Previous studies have demonstrated that TILs are associated with the prognosis of patients with CRC, and are also considered to be an important predictor of therapeutic 
A
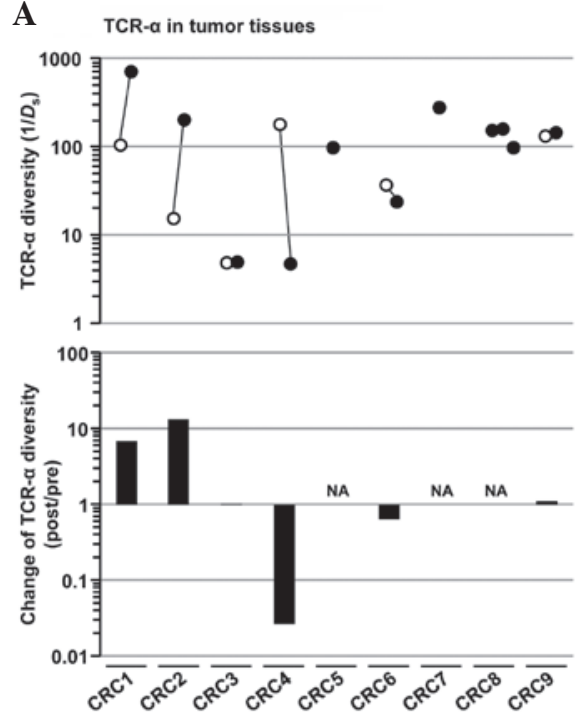

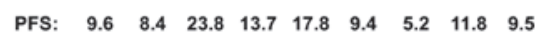
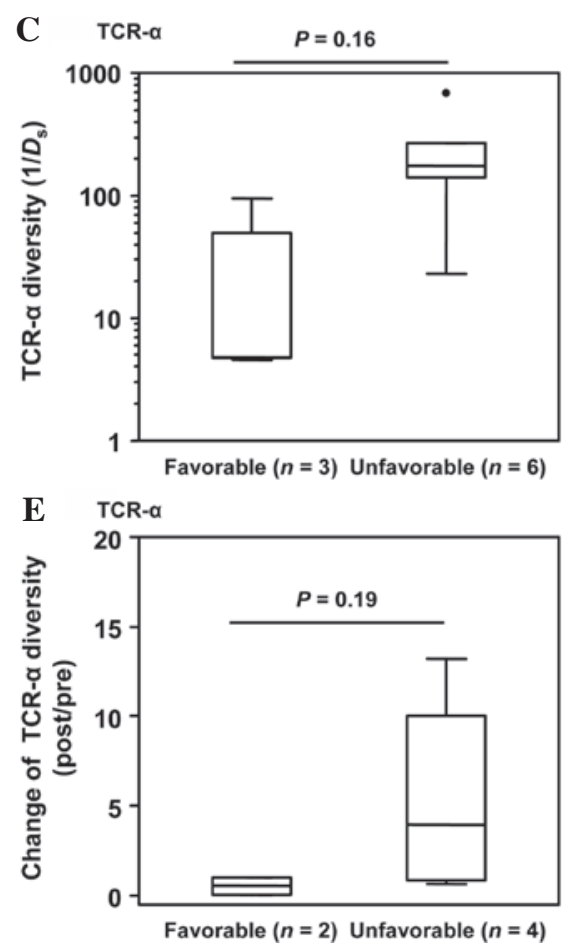

B
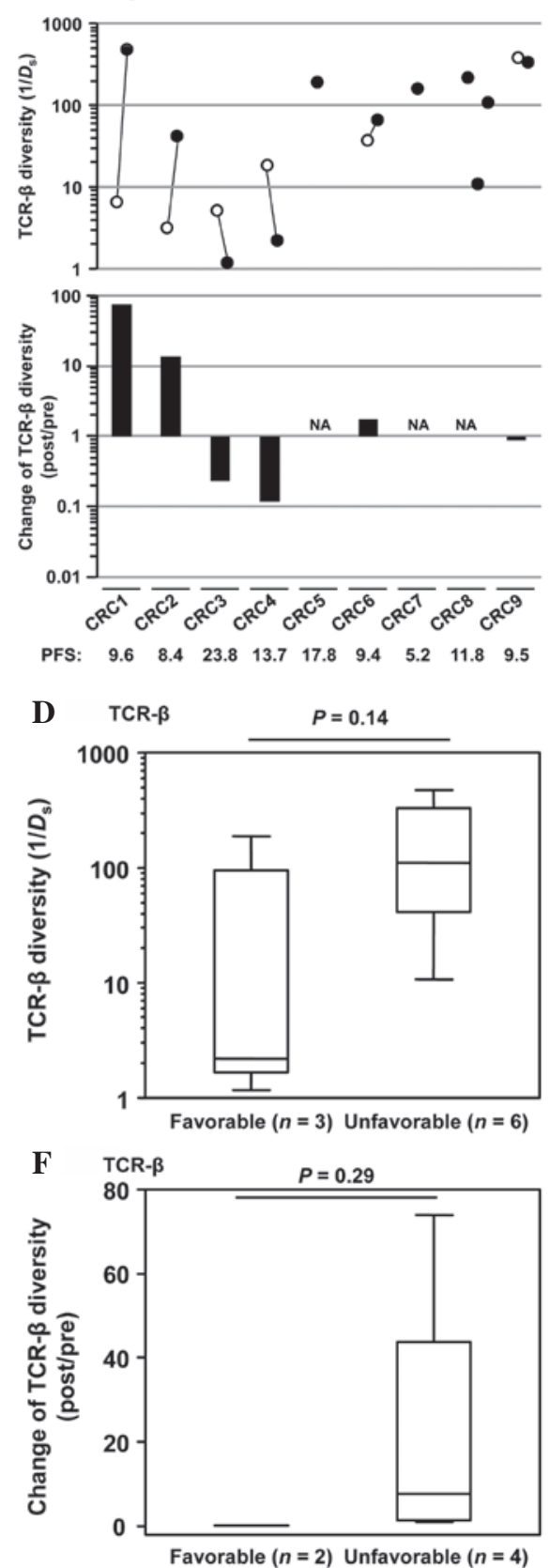

Figure 2. Association between TCR diversity in tumor tissues and clinical outcome of 9 patients with CRC. Changes in (A) TCR- $\alpha$ and (B) TCR- $\beta$ diversity during treatment in 9 patients with CRC. Open circles and closed circles indicate the diversity in tumor tissues at pre- and post-treatment, respectively. Comparison of (C) TCR- $\alpha$ and (D) TCR- $\beta$ diversity in post-treatment tumor tissues between the favorable ( $n=3$ ) and unfavorable $(n=6)$ groups. The horizontal line indicates the median concentration, the box covers the $25-75$ percentiles and the maximum length of each whisker is 1.5 times of the interquartile range. Each dot outside the whiskers indicates an outlier. Comparison of the ratio of (E) TCR- $\alpha$ and (F) TCR- $\beta$ diversity between favorable ( $n=2$; CRC 3 and CRC4) and unfavorable ( $n=4$; CRC1, CRC2, CRC6 and CRC9) groups. The horizontal line indicates the median concentration, the box covers the 25-75 percentiles and the maximum length of each whisker is 1.5 times the interquartile range. CRC, colorectal cancer; TCR, T cell receptor; $1 / D_{s}$, inverse Simpson's diversity index; PFS, progression-free survival.

responses (3-7). However, to the best of our knowledge, no previous studies have investigated the clonality or functionality of the $\mathrm{T}$ cells. In the present study, next-generation sequencers were used to comprehensively analyze the TCR repertoire in the tissue and blood samples obtained from advanced CRC patients that had been treated with cancer peptide vaccines in combination with oxaliplatin-based chemotherapy, and it was confirmed that the $\mathrm{T}$ cell repertoires in tissues varied from the $\mathrm{T}$ cell repertoires in blood samples, as previously described (22-27). Although previous studies only analyzed TCR clonotypes.

The majority of the enriched TCR sequences observed in pre-treatment tumor tissues were undetectable in the blood samples, but several clones enriched in post-treatment tumor tissues were detectable in the blood at a low frequency of $0.1-0.5 \%$. These common $\mathrm{T}$ cell populations in the post-treatment tissues and blood samples may possibly be activated in the lymph nodes near the vaccine injection sites 
Table I. Characteristics of patients with CRC and time points of sample collection.

\begin{tabular}{|c|c|c|c|c|c|c|c|}
\hline Patient & $\begin{array}{l}\text { Age, } \\
\text { years }\end{array}$ & Gender & Response & $\begin{array}{l}\text { Progression-free } \\
\text { survival, months }\end{array}$ & Outcome & $\begin{array}{l}\text { Time of tumor collection, } \\
\text { type }\left(\text { weeks }{ }^{\mathrm{a}}\right)\end{array}$ & $\begin{array}{c}\text { Time of blood } \\
\text { collection, weeks }\end{array}$ \\
\hline CRC1 & 55 & Male & PR & 9.6 & Succumbed & Colon (-6), liver (83) & $0,4,8,35,59$ \\
\hline CRC2 & 47 & Female & SD & 8.4 & Succumbed & Colon (-79), pancreas (17) & $0,4,35$ \\
\hline CRC3 & 66 & Male & SD & 23.8 & Alive & Colon (-142), lung (97) & $0,4,12,35,87$ \\
\hline CRC4 & 61 & Male & PR & 13.7 & Alive & Colon $(-5)$, liver $(63)$ & $0,4,19,35,59$ \\
\hline CRC5 & 36 & Male & PR & 17.8 & Alive & Colon (61) & $0,4,19,35$ \\
\hline CRC6 & 47 & Female & PR & 9.4 & Alive & Colon (-4), liver (39) & $0,5,19,35,77$ \\
\hline CRC7 & 65 & Female & PR & 5.2 & Succumbed & Colon (17) & $0,4,8,19$ \\
\hline CRC8 & 42 & Male & PR & 11.8 & Alive & Colon (27), lung (27), pelvis (55) & $0,4,8,23,44$ \\
\hline CRC9 & 69 & Female & PR & 9.5 & Alive & Colon (-3), small intestine (33) & $0,4,19$ \\
\hline
\end{tabular}

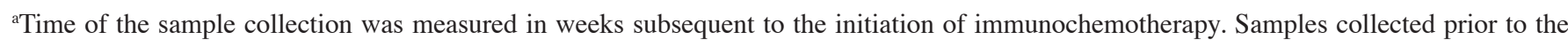
innitiation of immunochemotherapy are indicated by a negative number. CRC, colorectal cancer; PR, partial response; SD, stable disease.

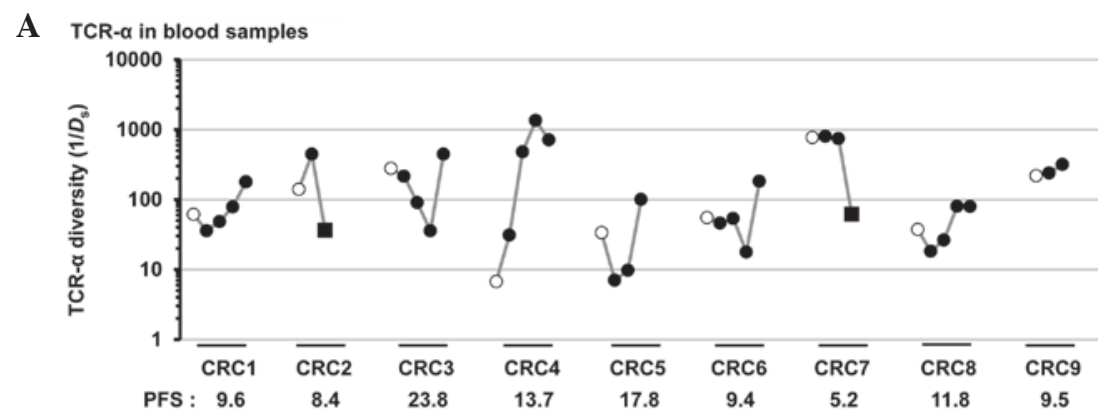

B TCR- $\beta$ in blood samples

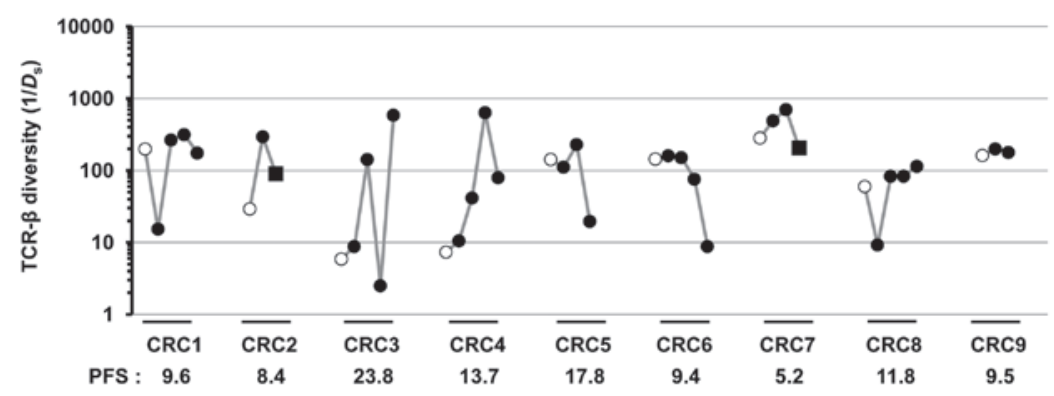

Figure 3. Diversity of the TCR clonotype in blood from 9 patients with CRC. (A) TCR- $\alpha$ and (B) TCR- $\beta$ diversity in blood samples at multiple time points in 9 patients with CRC. Open circles, closed circles and closed squares indicate the time points of pre-treatment, post-treatment and following surgery, respectively. TRC, T cell receptor; CRC, colorectal cancer; PFS, progression-free survival; $1 / D_{s}$, inverse Simpson's diversity index.

and then circulate in theblood. Certain T cell clones had accumulated and activated in the tumor lesions.

In addition, $\mathrm{T}$ cell repertoires were extremely different in the pre- and post-treatment tumor tissues, and in the primary and metastatic tumor tissues, indicating that the immune environment of cancer tissues changes markedly. In particular, the lack of a common clonotype between primary and lung metastatic tumors in patient $\mathrm{CRC} 8$, which were surgically resected at the same time, indicated the huge variation in the immune microenvironment, possibly due to the tumor heterogeneity or 'seed-soil' association, or a combination of the two.

For the evaluation of the vaccine-specific immune responses of patients, several currently-available approaches exist, including an enzyme-linked immunosorbent spot assay and an HLA-multimer assay (28). However, since an ex vivo expansion step of peripheral blood lymphocytes with antigen stimulation under certain cytokines is required, these methods are not suitable to quantitatively monitor patients' immune responses over time. Therefore, TCR sequencing with next generation sequencing was applied in order to monitor the immune responses. Increased TCR diversity in blood samples at an early time point, 8-19 weeks from the beginning of the treatment, was indicated to potentially reflect an improved immune response, in association with a better PFS (Fig. 3). A similar increase of TCR diversity was observed in another $T$ cell repertoire analysis in non-small cell lung cancer patients that were treated with cancer peptide vaccines (17). The increases of TCR diversity in blood samples may possibly be 
explained by the following mechanism: Cancer cells that are damaged by the treatment are likely to be phagocytosed by antigen-presenting cells, which present various cancer-specific antigens on their cell surface and eventually induce the activation of various $\mathrm{T}$ cell populations, including killer and helper $\mathrm{T}$ cells. TCR diversity in the blood samples may reflect the extent of the damage of cancer cells. Notably, the surgical resection of the tumors in two patients resulted in a significant decrease of TCR diversity in blood samples (Fig. 3) and the enhancement of clonal expansion of certain $\mathrm{T}$ cell populations in blood samples. These results may reflect the strong inflammatory reactions that occur following surgical treatment, or may reflect the immune reactions in the blood due to the transient increase of circulating tumor cells during the surgical procedure. TCR diversity scores in blood samples may be a predictive biomarker for the therapeutic response in advanced CRC patients. However, accumulating a large amount of information is clinically important, as TCR diversity is extremely complex and is affected by various environmental stimuli.

In summary, to the best of our knowledge, the present study is the first to deeply analyze the TCR- $\alpha$ and TCR- $\beta$ repertoires of tumor tissues and blood samples prior to and subsequent to immunotherapy in combination with chemotherapy in patients with advanced CRC. Although the significance of the present results is limited due to the small sample size, $\mathrm{T}$ cell populations in tumor tissues prior to the treatment were indicated to be almost entirely different from those in blood, but demonstrate commonality subsequent to vaccine treatment. The decrease of TCR diversity in the tumor tissues during the treatment may also be associated with a longer PFS. These results suggest that TCR diversity scores in the tissues may be a useful predictive biomarker for the therapeutic effect of immunochemotherapy for advanced CRC patients.

\section{Acknowledgements}

The present study was performed as part of the Project for Development of Innovative Research on Cancer Therapeutics and the Japan Agency for Medical Research and Development. The super-computing resource was provided by the Human Genome Center, Institute of Medical Science, University of Tokyo (http://sc.hgc.jp/shirokane.html).

\section{References}

1. Zitvogel L, Kepp O and Kroemer G: Immune parameters affecting the efficacy of chemotherapeutic regimens. Nat Rev Clin Oncol 8: 151-160, 2011.

2. Zitvogel L, Galluzzi L, Smyth MJ and Kroemer G: Mechanism of action of conventional and targeted anticancer therapies: Reinstating immunosurveillance. Immunity 39: 74-88, 2013.

3. Pagès F, Berger A, Camus M, Sanchez-Cabo F, Costes A, Molidor R, Mlecnik B, Kirilovsky A, Nilsson M, Damotte D, et al: Effector memory $\mathrm{T}$ cells, early metastasis and survival in colorectal cancer. N Engl J Med 353: 2654-2666, 2005.

4. Galon J, Costes A, Sanchez-Cabo F, et al: Type, density and location of immune cells within human colorectal tumors predict clinical outcome. Science 313: 1960-1964, 2006.

5. Laghi L, Bianchi P, Miranda E, et al: CD3+ cells at the invasive margin of deeply invading (pT3-T4) colorectal cancer and risk of post-surgical metastasis: A longitudinal study. Lancet Oncol 10 877-884, 2009.

6. Fridman WH, Pagès F, Sautes-Fridman C and Galon J: The immune contexture in human tumours: Impact on clinical outcome. Nat Rev Cancer 12: 298-306, 2012.
7. Yasuda K, Nirei T, Sunami E, Nagawa H and Kitayama J: Density of CD4(+) and CD8(+) T lymphocytes in biopsy samples can be a predictor of pathological response to chemoradiotherapy (CRT) for rectal cancer. Radiat Oncol 6: 49, 2011.

8. Rosenberg SA, Yang JC and Restifo NP: Cancer immunotherapy: Moving beyond current vaccines. Nat Med 10: 909-915, 2004.

9. Gajewski TF, Schreiber H and Fu YX: Innate and adaptive immune cells in the tumor microenvironment. Nat Immunol 14: 1014-1022, 2013.

10. Poggi A, Musso A, Dapino I and Zocchi MR: Mechanisms of tumor escape from immune system: Role of mesenchymal stromal cells. Immunol Lett 159: 55-72, 2014.

11. Janeway CA Jr, Travers P, Walport $M$ and Shlomchik MJ: Immunobiology: The Immune System in Health and Disease. 5th edition. Garland Science, New York, 2001.

12. Morris GP and Allen PM: How the TCR balances sensitivity and specificity for the recognition of self and pathogens. Nat Immunol 13: 121-128, 2012.

13. Arstila TP, Casrouge A, Baron V, Even J, Kanellopoulos J and Kourilsky P: A direct estimate of the human alphabeta $\mathrm{T}$ cell receptor diversity. Science 286: 958-961, 1999.

14. Arstila TP, Casrouge A, Baron V, Even J, Kanellopoulos J and Kourilsky P: Diversity of human alpha beta $\mathrm{T}$ cell receptors. Science 288: 1135, 2000.

15. Thor Straten P, Schrama D, Andersen MH and Becker JC: T-cell clonotypes in cancer. J Transl Med 2: 11, 2004.

16. Hazama S, Nakamura Y, Tanaka H, Hirakawa K, Tahara K, Shimizu R, Ozasa H, Etoh R, Sugiura F, Okuno K, et al: A phase II study of five peptides combination with oxaliplatin-based chemotherapy as a first-line therapy for advanced colorectal cancer (FXV study). J Transl Med 12: 108, 2014.

17. Fang H, Yamaguchi R, Liu X, Daigo Y, Yew PY, Tanikawa C, Matsuda K, Imoto S, Miyano S and Nakamura Y: Quantitative $\mathrm{T}$ cell repertoire analysis by deep cDNA sequencing of T cell receptor $\alpha$ and $\beta$ chains using next-generation sequencing (NGS). Oncoimmunology 3: e968467, 2015.

18. Giudicelli V, Chaume D and Lefranc MP: IMGT/GENE-DB: A comprehensive database for human and mouse immunoglobulin and T cell receptor genes. Nucleic Acids Res 33: D256-D261, 2005.

19. Langmead B and Salzberg SL: Fast gapped-read alignment with Bowtie 2: Nat Methods 9: 357-359, 2012.

20. Yousfi Monod M, Giudicelli V, Chaume D and Lefranc MP: IMGT/JunctionAnalysis: The first tool for the analysis of the immunoglobulin and $\mathrm{T}$ cell receptor complex V-J and V-D-J JUNCTIONs. Bioinformatics 20 (Suppl 1): S379-S385, 2004.

21. Venturi V,Kedzierska K, Turner SJ, Doherty PC and Davenport MP: Methods for comparing the diversity of samples of the T cell receptor repertoire. J Immunol Methods 321: 182-195, 2007.

22. Ochsenreither S, Fusi A, Wojtke S, Busse A, Nüssler NC, Thiel E, Keilholz U and Nagorsen D: Comparison of T-cell receptor repertoire restriction in blood and tumor tissue of colorectal cancer patients. J Transl Med 8: 35, 2010.

23. Sherwood AM, Emerson RO, Scherer D, Habermann N, Buck K, Staffa J, Desmarais C, Halama N, Jaeger D, Schirmacher P, et al: Tumor-infiltrating lymphocytes in colorectal tumors display a diversity of $\mathrm{T}$ cell receptor sequences that differ from the $\mathrm{T}$ cells in adjacent mucosal tissue. Cancer Immunol Immunother 62: 1453-1461, 2013.

24. Luo W, Liao WJ, Huang YT, Shi M, Zhang Y, Wen Q, Zhou MQ and Ma L: Normalization of T cell receptor repertoire diversity in patients with advanced colorectal cancer who responded to chemotherapy. Cancer Sci 102: 706-712, 2011.

25. Zhou J, Ma R, Luo R, Sun Y, He X, Sun W, Tang W and Yao X: Primary exploration of CDR3 spectratyping and molecular features of TCR $\beta$ chain in the peripheral blood and tissue of patients with colorectal carcinoma. Cancer Epidemiol 34: 733-740, 2010.

26. Mlecnik B, Tosolini M, Kirilovsky A, Berger A, Bindea G, Meatchi T, Bruneval P, Trajanoski Z, Fridman WH, Pagès F and Galon J: Histopathologic-based prognostic factors of colorectal cancers are associated with the state of the local immune reaction. J Clin Oncol 29: 610-618, 2011.

27. Nagorsen D, Keilholz U, Rivoltini L, Schmittel A, Letsch A, Asemissen AM, Berger G, Buhr HJ, Thiel E and Scheibenbogen C: Natural T-cell response against MHC class I epitopes of epithelial cell adhesion molecule, her-2/neu and carcinoembryonic antigen in patients with colorectal cancer. Cancer Res 60: 4850-4854, 2000.

28. Czerkinsky C, Andersson G, Ekre HP, et al: Reverse ELISPOT assay for clonal analysis of cytokine production. I. Enumeration of gamma-interferon-secreting cells. J Immunol Methods 110: 29-36, 1988. 